\title{
MULTILINEAR VARIANTS OF GROTHENDIECK'S COMPOSITION THEOREM
}

\author{
DUMITRU POPA
}

Abstract. In this paper we prove two multilinear variants of Grothendieck's composition theorem. We give examples which show that other natural multilinear variants of Grothendieck's composition theorem are not necessarily true. In addition we prove a new multilinear variant of Grothendieck's composition theorem.

Mathematics subject classification (2010): Primary 47H60; Secondary 46B25, 46C99.

Keywords and phrases: $p$-summing, multilinear operators, multiple summing, Hilbert-Schmidt, nuclear operators.

\section{REFERENCES}

[1] G. BADEA, D. Popa, Hilbert-Schmidt and multiple summing operators, Collect. Math. 63, No. 2 (2012), 181-194.

[2] F. Bombal, D. Pérez-García, I. Villanueva, Multilinear extensions of Grothendieck's theorem, Quart. J. Math. 55 (2004), 441-450.

[3] F. Cobos, T. KÜhn, J. Peetre, Schatten-von Neumann classes of multilinear forms, Duke Math. J. 65, No. 1 (1992), 121-156.

[4] A. Defant, K. Floret, Tensor norms and operator ideals, North-Holland, Math. Studies, 176, 1993.

[5] A. Defant, D. Popa, U. Schwarting, Coordinatewise multiple summing operators in Banach spaces, Journ. Funct. Anal. 259, No. 1 (2010), 220-242.

[6] J. Diestel, H. Jarchow, A. Tonge, Absolutely Summing Operators, Cambridge Stud. Adv. Math. 43, Cambridge University Press, 1995.

[7] S. DineEn, Complex analysis in locally convex spaces, North-Holland Math. Studies, 57, 1981.

[8] M. C. MATOS, Fully absolutely summing and Hilbert-Schmidt multilinear mappings, Collect. Math. 54 (2003), 111-136.

[9] D. PÉREZ-GARCÍA, The inclusion theorem for multiple summing operators, Studia Math. 165, No. 3 (2004), 275-290.

[10] D. PÉreZ-García, I. VillaneVa, Multiple summing operators on $C(K)$ spaces, Arkiv for Mat. 42 (2004), 153-171.

[11] D. Pérez-García, I. Villanueva, A Composition Theorem for Multiple Summing Operators, Monatsh. Math. 146, No. 3 (2005), 257-261.

[12] A. Pietsch, Operator ideals, Veb Deutscher Verlag der Wiss., Berlin, 1978; North Holland, 1980.

[13] D. POPA, Nuclear multilinear operators with respect to a partition, Rendiconti del Circolo Matematico di Palermo, 61, No. 3 (2012), 307-319.

[14] M. S. Ramanujan, E. SCHOCK, Operator ideals and spaces of bilinear operators, Linear and Multilinear Algebra 18 (1985), 307-318. 